$\begin{array}{lllll}\text { Revista latinoamericana de Medicina Conductual } & \text { Vol } 1 & \text { Num } 1 & 8-2010 & \text { pp } 13-20\end{array}$

\title{
ANÁlISIS DE MOdELOS CONCEPTUALES EMPLEADOS EN Medicina Conductual
}

María del Rocío Hernández-Pozo

Proyecto de Investigación Aprendizaje Humano, FES IZTACALA Universidad Nacional Autónoma de México

\section{Resumen}

De acuerdo con Dekker (2008) se esta observando un uso cada vez mas elaborado de teorías en el área de la Medicina Conductual, tendencia que mejora la comprensión de los hallazgos empíricos, a pesar de ello, la mayoría de las veces los enfoques teóricos no generan diferencias metodológicas acordes, ni tampoco diferencias en el tipo de intervenciones. Adicionalmente, el lenguaje natural en que se expresan estos modelos puede oscurecer los planteamientos, - hacer parecer similares modelos que no guardan semejanza entre si. En este escrito se emplea el lenguaje contingencial propuesto por Mechner (2008) como un instrumento para comparar instancias de planteamientos teóricos diferente. Se presenta la descripción de una investigación representativa de la teoría de estrés y regulación emocional (Brown, Katzel, Neumann, Maier \& Waldstein, 2007); un estudio asociado a la teoría de conducta saludable, salud y enfermedad (van Dijk, Veenhof, Spreeuwenberg, Coene, Burger, et al., 2010) y un ejemplo de la teoría de la personalidad y su influencia en la salud (Denollet \& De Vries, 2006). Se tradujeron los modelos a diagramas contingenciales para esclarecer el orden de sus intereses, comparar niveles de complejidad, y subrayar algunas consecuencias prácticas de cada enfoque. Un examen visual de los diagramas, puso en evidencia las diferencias entre enfoques y llevó a la conclusión que los modelos seleccionados más que excluyentes, son complementarios.

Palabras clave: Análisis contingencial, modelos, medicina conductual, Mechner, notación simbólica, teorías, traducción.
According to Dekker (2008) the field of Behavioral Medicine is witnessing a more elaborated use of theories to further comprehension of empirical findings, however most of the times the differences among these models are elusive, specially in terms of the methodological consequences for acceptable research to elucidate central assumptions of such models. The contingency analysis of behavior language elaborated by Mechner (2008) is proposed as a methodological tool to compare such models, by means of translating representative exemplars of three theoretical approaches. Diagrams of the stress and emotional regulation theory (Brown, Katzel, Neumann, Maier \& Waldstein, 2007), the theory on health behavior, health and disease (van Dijk, Veenhof, Spreeuwenberg, Coene, Burger, et al., 2010) and the theory of personality and health (Denollet \& De Vries, 2006) are presented in detail in order to unravel the hierarchy of their interests, to compare levels of complexity, and to highlight some practical consequences of each approach. A visual inspection of the diagrams made apparent differences among the targeted models and lead to conclude that rather than being excluding, they complement each other.

Key words: Contingency analysis, models, behavioral medicine, symbolic notation, theories, translation. 


\section{Introducción}

El empleo de modelos en la investigación sobre comportamiento saludable como lo señala Dekker (2008) es muestra de un grado de madurez alcanzado por las disciplinas que constituyen el área de la Medicina Conductual. Someter a escrutinio hipótesis sobre cambios en un fenónemo bajo estudio, como consecuencia de la selección de variables que lo afecten y poder generar predicciones cualitativas y cuantitativas al respecto, evidencian que los analistas e investigadores conocen a fondo dicho fenómeno, y están generando conocimiento acumulativo sobre el mismo, que permitirá avanzar a la disciplina y esclarecer problemas que antes eludían su comprensión.

Existen varios criterios de utilidad para los modelos conceptuales en el quehacer científico, particularmente en el trabajo interdisciplinario que involucra el área de salud. Un modelo es útil en la medida en que propone relaciones direccionales entre variables, que en su conjunto dan cuenta o predicen la ocurrencia de un fenómeno complejo. Otro criterio de utilidad para un modelo es que sugiera formas particulares de medición de las variables y que transparente los procedimientos que al adoptarse, pueden servir para apoyar o desechar dicha propuesta. Si como generalmente ocurre, un fenómeno de interés puede describirse de diferentes maneras, o diagramas de relaciones hipotéticas entre variables, entonces, podríamos concluir, que el mejor modelo sería aquel que represente a las variables en juego y sus interrelaciones, de la manera mas simple y con el mayor poder predictivo, bajo diferentes condiciones. Si aceptamos este razonamiento, podríamos concluir que un análisis comparativo de diferentes modelos en Medicina Conductual, puede ayudarnos a elegir la propuesta de relaciones hipotéticas entre variables que mejor resuma, prediga y sea contrastable, en función de sus supuestos, donde la parsimonia y el poder predictivo ocuparían el fiel de la balanza.

El área de estudio de cambio en el comportamiento en general cuenta con un mosaico de teorías y modelos desarrollados en los últimos cien años, que incluyen: al condicionamiento clásico de Pavlov, el condicionamiento operante de Skinner, la desensibilización sistemática de Wolpe y la terapia cognitiva conductual de Beck y Ellis. Entre los desarrollos posteriores y extensiones hacia el campo de la salud se encuentran: el modelo de creencias en la salud de Rosenstock, la teoría de la acción razonada de Fishbein, el modelo transaccional de Folkman y Lazarus, la teoría de la conducta planeada de Ajzen y el modelo transteorético de Prochaska \& Di Clemente (Marks, 2009).

Otros autores (Michie, Johnston, Francis, Hardeman \& Eccles, 2008) en sus consideraciones sobre las teorías de mayor influencia en investigación en salud, se enfocan a un abanico mas exclusivo, que incluye solamente a las de la teoría de la conducta planeada de Ajzen, la teoría cognitiva social de Bandura y la teoría del aprendizaje operante de Skinner.

Se eligió la propuesta de Dekker, por encima de otras propuestas con un énfasis diferente (Collins, Hewson, Munger \& Wade, 2010), debido al interés teórico de la propuesta y por el papel central que desempeña ese autor como vocero de una de las revistas de mayor prestigio en el área, la Revista Internacional de Medicina Conductual.

El problema central que enfrenta un proyecto que intente comparar diferentes modelos en una área particular de conocimiento, es que estos con frecuencia son ideados a partir de aproximaciones diversas, conceptuales y metodológicas, de modo que hacen prácticamente imposible la comparación, ya que no hay elementos en común a partir de los cuales proceder a aquilatarlos. Para poder proceder a un análisis comparativo de propuestas teórico-procedurales, el primer paso a dar consiste en emplear una herramienta que traduzca lo incomesurable a un sistema que use elementos de análisis y una óptica con un denominar común, o como lo ha señalado Marks (2009), una forma de etiquetar o describir una intervención psicológica, independientemente de la orientación teórica, de manera que se pueda comunicar a otros de forma precisa.

El análisis contingencial propuesto por Mechner (2008) es una herramienta que permite traducir los elementos centrales de cualquier interacción psicológica compleja, a partir de los elementos de los que es función, transparentando las relaciones condicionales clave que nos permitan hacer predicciones y corroborarlas, contrastar hipótesis alternas, o planear intervenciones, a partir de elementos críticos que mantienen por ejemplo, patrones de comportamiento beneficioso o nocivo para la salud individual, o de un grupo de personas.

La propuesta de Mechner (2008) consiste en el empleo de símbolos, muy similares a los que se emplean en lógica matemática, pero pensados dentro de un lenguaje diseñado ex profeso para analizar comportamiento de organismos que aprenden y que modifican sus acciones, en función de las consecuencias positivas o negativas que producen y regulan. Este lenguaje contingencial consta de acciones, agentes, 


\section{Resultados}

verbos, modificadores y relaciones entre ellos, que se denotan a partir de letras mayúsculas y minúsculas, signos como más, menos, tildes, equis, signo de interrogación, que pueden adoptar cuatro posiciones (superior izquierda, inferior izquierda, superior derecha e inferior derecha) a partir del elemento al que modifican. La ventaja de este sistema de notación diagramática, que es el eje del lenguaje contingencial, es que permite, mediante el mismo sistema de relaciones, describir de manera precisa un sinnúmero de fenómenos que pertenecen a la misma clase, debido al tipo de relaciones y elementos que los definen.

El simple examen visual de un diagrama de este tipo, permite determinar el grado de complejidad de la propuesta del investigador que aborda el estudio de un fenómeno particular, posibilita hacer comparaciones entre diagramas diferentes y permite representar el orden de relaciones condicionales que propone el modelo, y detectar el o los elementos cruciales, cuya presencia o ausencia podría cambiar radicalmente al fenómeno. En síntesis la notación contingencial de Mechner, es un lenguaje que provee un medio de comparación funcional de las propuestas conceptuales y programáticas de los investigadores.

El presente estudio tiene como propósito identificar los elementos definitorios de tres planteamientos teóricos señalados por Dekker (2008) en el campo de la Medicina Conductual, traducir sus componentes a un lenguaje contingencial y compararlos entre sí desde esa óptica.

\section{Método}

Se seleccionó un artículo representativo de tres aproximaciones teóricas de las mencionadas por Dekker (2008) como representativas del área de la Medicina Conductual, a saber: a) la teoría de estrés y regulación emocional; b) la teoría de conducta saludable, salud y enfermedad, y c) de la teoría de la personalidad y su influencia en la salud. Posteriormente a partir de los artículos seleccionados, se identificaron los elementos centrales de cada propuesta conceptual, se propuso una traducción contingencial de los mismos a partir del lenguaje simbólico desarrollado por Mechner (2008) y finalmente se realizó un análisis comparativo basado en la inspección visual de los mismos diagramas así generados.

\section{Teoría de estrés y regulación emocional}

La investigación seleccionada para representar esta aproximación fue el estudio de Brown, Katzel, Neumann, Maier \& Waldstein (2007). En general esta orientación teórica mantiene que la respuesta de estrés depende de un estímulo estresor externo, de la reacción ante el estresor y de variables intermedias (Dekker, 2008). En el estudio en cuestión la reactividad cardiovascular de 42 personas con isquemia silenciosa inducida por ejercicio, se comparó con la de un grupo control, ante tres condiciones de estrés: a) recuerdo de una situación de enojo, b) hablar en público y c) resolver una tarea matemática bajo presión. El grupo experimental mostró frecuencia cardiaca significativamente superior a la del grupo control, solo en la condición de hablar en público, sin mostrarse cambios en tensión arterial. Ninguna de las otras dos condiciones para generar estrés produjo su objetivo, en ninguno de los grupos.

El modelo contingencial simplificado de la investigación descrita consiste en dos opciones, en la primera dada una condición de recuerdo de una situación de enojo o de resolver una tarea matemática bajo presión $\left(C_{1}\right)$ el agente (a) que en este caso corresponde al grupo experimental, reacciona (A) de manera normal, lo cual produce una condición fisiológica sin estrés $\left(C_{2}\right)$, que en este caso tiene una valencia positiva para el agente (a). En la segunda opción, dada la condición $\left(C_{3}\right)$, que consiste en hablar en público, el agente $(a)$ reacciona $(A)$ y esa reacción produce una condición $\left(C_{4}\right)$ con valencia negativa para el agente $a$, que consiste en una alteración de la frecuencia cardiaca en sentido de aumento de esta.

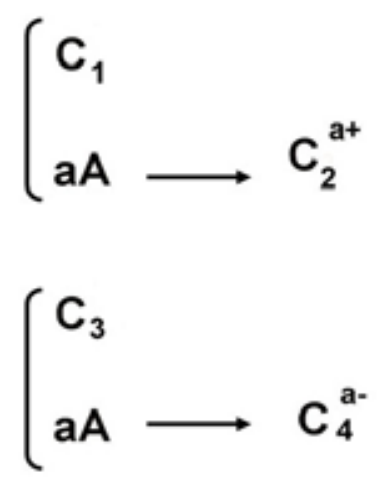

Figura 1. Notación contingencial del modelo de estrés y regulación emocional de Brown, Katzel, Neumann, Maier \& Waldstein (2007) 
En este caso, el diagrama contingencial del modelo, es muy simple, señalando tan solo una situación con dos opciones, una con un resultado negativo para la salud, y otra sin ese resultado, tal como se representa en la Figura 1. Por otro lado el esquema a pesar de su simplicidad resalta desde un punto de vista terapéutico, dos aspectos centrales. El primero, que sería ventajoso para el agente a, que aprendiera a discriminar entre condiciones inocuas $\left(C_{1}\right)$ y adversas $\left(C_{3}\right)$, de modo que evite éstas últimas y disminuya la probabilidad de enfrentar sus consecuencias negativas $\left(C_{4}\right)$, y segundo lugar, que una vez identificadas las condiciones adversas $\left(C_{3}\right)$, si la situación no permite evitarlas, sería ventajoso para el paciente, que aprendiera a lidiar con ellas de manera que siga el menor daño posible $\left(\mathrm{C}_{4}\right)$.

Teoría de conducta saludable, salud y enfermedad Este tipo de modelo aborda el estudio de los mecanismos que dan cuenta de la relación entre comportamientos asociados con la salud y el curso de los procesos de salud y enfermedad (Dekker, 2008). Como ejemplo de esta aproximación, se seleccionó una investigación realizada por van Dijk, Veenhof, Spreeuwenberg, Coene, Burger, et al. (2010). Estos au- tores reportan los cambios experimentados por 237 pacientes mayores con problemas de osteoartritis en cadera o rodilla, en el curso de un período de observación de 3 años, con medidas repetidas anuales del curso de las limitaciones físicas que experimentaron (via un cuestionario de cambios percibidos por los pacientes, auto-reporte de limitaciones y observación), a partir de tres factores predictivos que incluyeron: funciones corporales (dolor, fuerza muscular, rango de movimiento), comorbilidad (puntaje en una escala diseñada ex profeso, que asignaba pesos a diferentes malestares y problemas de salud que experimentaba el paciente simultáneamente a su padecimiento) y funciones cognoscitivas (declinación cognitiva, memoria y atención). Los autores del estudio reportan estabilidad en la limitación de las actividades a nivel grupal, durante el seguimiento de tres años, aunque a nivel individual se registraron declives significativos predecibles a partir de la disminución durante el primer año del seguimiento, de las tres funciones corporales bajo estudio, por el aumento del puntaje de comorbilidad, y en menor medida, por el decremento en las capacidades cognitivas.

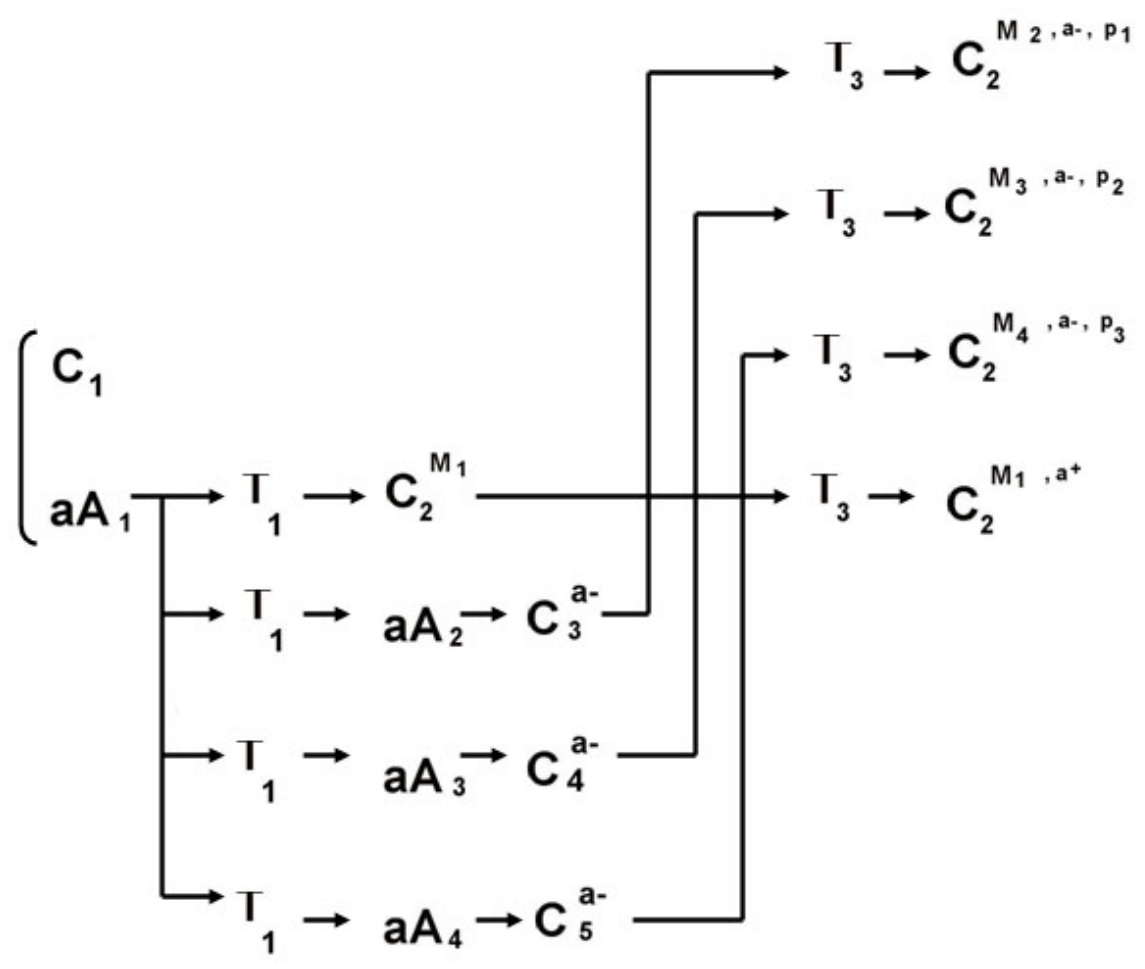

Figura 2. Notación contingencial de un ejemplo del modelo de conducta saludable, salud y enfermedad de van Dijk, Veenhof, Spreeuwenberg, Coene, Burger, et al. (2010) 
El diagrama contingencial de la investigación anterior se presenta en la Figura 2, que describe que dada la condición de osteoartritis $\left(C_{1}\right)$, el agente (a) realiza sus actividades cotidianas $\left(A_{1}\right)$ y al cabo de un año $\left(T_{1}\right)$ presenta limitaciones físicas $\left(C_{2}\right)$ de cierta magnitud $\left(M_{1}\right)$, y posteriormente al cabo de tres años $\left(T_{3}\right)$ sus limitaciones físicas $\left(C_{2}\right)$ permanecen sin cambio, esto es, son de la misma magnitud $\left(M_{1}\right)$, lo cual es positivo para el agente $(a+)$. Una segunda secuencia de sucesos sería si el agente (a) experimentara algún cambio en su funcionamiento corporal, como aumento del dolor en cadera o rodilla, disminución de la fuerza muscular, o disminución en el rango de movimiento $\left(A_{2}\right)$, que produciría una consecuencia $\left(C_{3}\right)$ con valencia negativa para el agente (a-), entonces al cabo de tres años $\left(T_{3}\right)$ las limitaciones físicas $\left(C_{2}\right)$ tendrían una magnitud mayor $\left(M_{2}\right)$, con una determinada probabilidad $\left(p_{1}\right)$, lo cual sería negativo para el agente (a-). Una tercera cadena de sucesos tendría lugar si al cabo de un año, el agente (a) se enfermara de algún otro padecimiento $\left(A_{3}\right)$, además de la osteoartritis de rodilla/cadera, lo cual produciría una consecuencia $\left(\mathrm{C}_{4}\right)$ negativa para el paciente (a-), y al cabo de tres años $\left(T_{3}\right)$ las limitaciones físicas $\left(C_{2}\right)$ tendrían una magnitud de un valor mayor $\left(M_{3}\right)$, con una probabilidad determinada $\left(\mathrm{p}_{2}\right)$. Finalmente un cuarto derrotero podría ocurrir si después de un año, el agente (a) sufriera de trastornos cognitivos, deficiencias en el recuerdo o fallas de atención $\left(A_{4}\right)$, lo que generaría una consecuencia $\left(C_{5}\right)$ negativa para el paciente (a-), que produciría que después de tres años $\left(T_{3}\right)$ las limitaciones físicas $\left(C_{2}\right)$ del paciente, tendrían una magnitud de un valor determinado $\left(M_{4}\right)$, con una probabilidad específica $\left(p_{3}\right)$, lo cual tendría una valencia negativa para el agente $a$.

En este caso la observación de tipo terapéutico, que se deriva del diagrama, sería cuidar a toda costa que el paciente durante el primer año $\left(T_{1}\right)$, no se involucre en las acciones $A_{2}$ y $A_{3}$, que son las que tienen el mayor costo asociado para la prognosis a los 3 años $\left(T_{3}\right)$, e investigar el tipo de acciones de vida normal $\left(A_{1}\right)$ que realizó el agente (a) que tuvieron que ver con la protección para la salud, en la forma de no sufrir un aumento en las limitaciones físicas al plazo de tres años que duró del estudio.

Teoría de la personalidad y su influencia en la salud El artículo que se eligió como representativo de una versión contemporánea de este enfoque fue el estudio de Denollet y De Vries (2006) sobre afecto positivo y negativo, en relación con la depresión, el estrés y la fatiga, que forma parte de un conjunto de inves- tigaciones que suponen que ciertas características de personalidad distresada ("D"), producto del efecto simultáneo del afecto negativo y la inhibición social, hacen vulnerables a los individuos a desarrollar padecimientos cardiovasculares (Denollet, 2005; Pedersen \& Denollet, 2006).

El estudio seleccionado presenta una propuesta específica que atribuye al modelo bifactorial de distrés, riesgos específicos para la salud. Denollet y De Vries (2006) señalan que mientras el afecto positivo está asociado de manera directa con la calidad de vida de la persona, el afecto negativo compromete la salud. El afecto negativo para estos autores, presenta dos vertientes, por un lado el afecto negativo pasivo (indisposición, desactivación, desgaste emocional), que se mide a través de la "escala global del estado de ánimo", GPS por sus siglas en inglés (Denollet, 1993), y el afecto negativo activo (intranquilidad, aprensión ansiosa, temor), valorado por el cuestionario denominado "programa de afecto positivo y negativo", PANAS por sus siglas en inglés (Watson, Clark \& Tellegen, 1988). La tesis de estos autores en resumen consiste en que atribuyen al estilo de responder con afecto negativo elevado y afecto positivo bajo, como patrón reactivo característico, una alta probabilidad de síntomas depresivos, fatiga y desgaste laboral, que en su conjunto producen consecuencias adversas para la salud, tales como susceptibilidad para desarrollar infecciones respiratorias, cáncer y accidentes cardiovasculares, en una especia de círculo vicioso que se retroalimenta.

En términos contingenciales, el modelo se traduce a una situación adversa inicial $\left(\mathrm{C}^{\mathrm{a}}\right)$, que en este caso consiste en una tendencia del agente (a) a actuar de cierta manera (A) que produce consecuencias que son predichas erróneamente por el agente (a) y percibidas erróneamente por el agente (a) como adversas, lo cual produce a su vez consecuencias con valencia negativa para el agente (a), que retroalimentan a la condición inicial adversa para el agente (a). Si la tendencia generalizada inicial del agente a $\left(\mathrm{C}^{\mathrm{a}-}\right)$, coincide con las acciones de otra persona, por ejemplo, el agente $b$, ocurre que las consecuencias del comportamiento del agente $b$, en presencia del agente a, son predichas y percibidas erróneamente por el agente a como con valencia negativa para el agente $a$, lo cual produce que el agente a, actúe de modo que éstas generan a su vez consecuencias de valencia negativa para $b$, siendo lo anterior una condición que evita que el agente b siga involucrándose en acciones que produzcan consecuencias con valencia positiva para $a, y$ mas aún, produce que el agente $b$ 
realice acciones con consecuencias negativas para a, lo cual retroalimenta la condición inicial con valencia negativa para el agente a $\left(\mathrm{C}^{\mathrm{a}}\right)$.

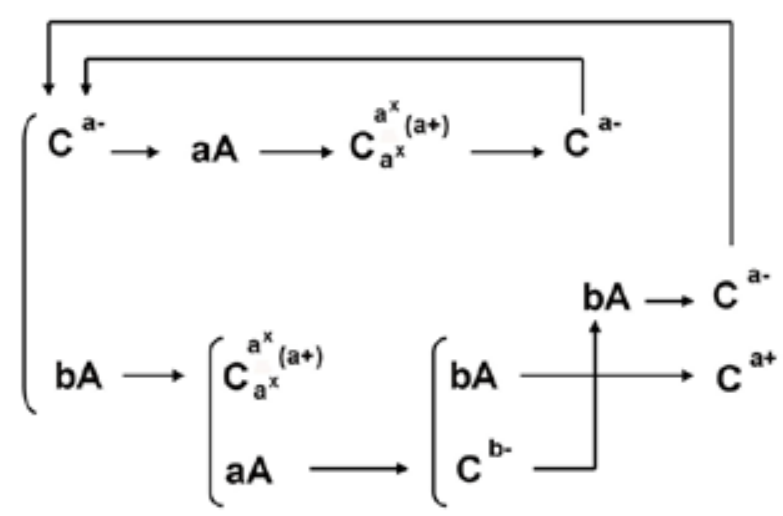

Figura 3. Notación contingencial del modelo de personalidad Tipo "D" de Denollet y De Vries, (2006)

El punto clave de intervención en este modelo, consiste en la condición inicial con valencia negativa para el agente a $\left(\mathrm{C}^{\mathrm{a}}\right)$, que sería el elemento a modificar, así como romper el ciclo de retroalimentación que mantiene la condición adversa como una constante.

La comparación de los tres diagramas, a partir de su inspección visual, lleva a derivar dos conclusiones, la primera, que existen diferencias en los niveles de complejidad de los mismos, siendo el primer modelo, el más simple y el segundo el más complejo, al involucrar magnitudes y probabilidades diferentes, asociadas a cada secuencia de acciones. Por otro lado, el tercer diagrama es el único que produce retroalimentación, a diferencia de los otros dos, pero debido al menor uso de modificadores, este último cae en términos comparativos, a un nivel medio.

La segunda conclusión que se deriva de la comparación entre modelos empleando este método, es que los diagramas no son excluyentes, sino que en principio, podrían ser complementarios, en el sentido de que cada uno se enfoca a un interés particular, sea éste: a) determinar lo que dispara o no una reacción nociva para la salud, b) los elementos que a mediano plazo predicen un deterioro mayor para pacientes con un problema crónico degenerativo, o c) verificar que ciertos patrones de comportamiento mantienen un círculo vicioso que compromete la salud.

Por otro lado, si bien los investigadores reportan el uso de instrumentos diferentes particulares a sus estudios, esa condición no necesariamente los hace diferentes entre sí, desde un punto de vista contin- gencial, por lo que se descarta como elemento dentro de este tipo de análisis comparativo. La representación del entramado contingencial de las tres investigaciones, permite comparar con el mismo rasero y lógica aproximaciones que de otro modo no tendrían elementos en común.

\section{Discusión}

Existen por supuesto múltiples criterios para escoger un modelo particular de investigación en Medicina Conductual, uno de los cuales puede ser suscribirse explícita o implícitamente a un compromiso teórico particular. Algunos de esos criterios implican el concepto de transparencia, con los elementos respectivos de claridad y profundidad en el detalle que se ofrece en la descripción, que juntos permiten la replicabilidad; además de la transparencia, existen otros criterios como la parsimonia o "limpieza de excesos", y la lucha contra la inercia (Marks, 2009).

Independientemente de consideraciones de filosofía y sociología de la ciencia, la adopción de modelos particulares en el quehacer de investigación en Medicina Conductual, puede ser auxiliada por herramientas como la que se presenta en esta investigación. En este sentido el lenguaje contingencial de Mechner constituye una estrategia prometedora, que brinda al estudioso, elementos de juicio valiosos desde un punto de vista de relaciones condicionales entre elementos, y minimiza las ambigüedades propias del lenguaje natural.

Un análisis de la traducción de los elementos centrales de las aproximaciones empleadas por los estudios en Medicina Conductual aquí abordados, sugiere, que mas que propuestas teóricas complejas, o modelos con pesos específicos predictivos en lo cualitativo o cuantitativo de los fenómenos de comportamiento y salud, los casos abordados son instancias simples que los investigadores enfatizan como parte de un esquema muchas veces implícito de su programa de investigación.

Mapear la metodología de intervención en salud, en función de las aproximaciones teóricas en que están fundamentadas, no es una tarea fácil, como lo demuestra el trabajo de algunos investigadores que han hecho intentos preliminares en ese sentido. Michie, Johnston, Francis, Hardeman y Eccles (2008) hicieron un primer intento exploratorio en el cual, cuatro expertos en el área, mapearon 137 técnicas que se emplean como procedimientos de cambio conductual, obteniendo un $75 \%$ de acuerdo en la asociación de esas técnicas con un constructo teórico 
específico. La tarea de vincular técnicas de intervención con marcos conceptuales particulares reviste dificultades, ya que los planteamientos conceptuales se han empleado mas que nada para describir fenómenos, y no tanto para seleccionar una metodología de intervención (Michie, Johnston, Francis, Hardeman \& Eccles, 2008). Aunado a lo anterior, con frecuencia técnicas eficientes surgidas dentro de un marco conceptual particular, son aplicadas desprovistas de los supuestos que las hicieron surgir, desde perspectivas teóricas diferentes y en algunos casos antagónicas a las que les dieron origen, siendo este el caso, muchas veces, sin que los investigadores estén conscientes de esos tipos de "préstamos" metodológicos.

El análisis contingencial de las instancias de "modelos" de investigación abordados en el presente estudio, está lejos de ser exhaustivo y más bien corresponde a un ejercicio que ejemplifica las bondades de mirar a través de una óptica contingencial, los reportes de investigación de algunas modelos que se publican en el área. Quedan por desarrollar traducciones de propuestas de modelos de investigación diferentes, como lo serían entre otros, la teoría de los determinantes contextuales del comportamiento saludable (Siegrist, Wahrendorf, Knesebeck, Jürges \& Börsch-Supan, 2006) y la teoría cognitiva social del comportamiento saludable (Schüz, Sniehotta, Mallach, Wiedemann \& Schwarzer, 2009), por mencionar tan solo a algunos. Modelos más completos, que ofrezcan predicciones cualitativas y cuantitativas relacionadas con el papel que juega el comportamiento en la recuperación de la salud y la evitación de la enfermedad, presentados en términos contingenciales, sería guías útiles para reflexionar sobre los caminos que se han explorado y las posibilidades nuevas de plantear su abordaje.

Este escrito aborda una forma particular de analizar modelos empleados en el área de Medicina Conductual, que permite tomar decisiones basadas en la claridad y parsimonia de la propuesta conceptual, a partir de las contingencias que implica cada aproximación al problema de investigación. Sin embargo, existen aspectos de orden sociológico que afectan el que determinados modelos tengan mayor impacto en épocas o regiones particulares; como lo señala Marks (2009), las teorías o "mitos" que integran sabiduría, conocimientos y sentido común, con frecuencia son el resultado de decisiones históricas, inspiradas por intereses externos al campo disciplinario. Si bien la reflexión de Marks es pertinente, no invalida el que los que nos dedicamos a la investigación en la Medicina Conductual, busquemos y construyamos crite- rios a partir de los cuales podamos entender nuestro trabajo y el trabajo de los demás. Este escrito tan solo es un primer paso en esa dirección.

\section{Referencias}

Brown, J. P., Katzel, L. I., Neumann, S. A., Maier, K. J., \&Waldstein, S. R. (2007). Silent myocardial ischemia and cardiovascular responses to anger provocation in older adults. International Journal of Behavioral Medicine, 14, 134-140.

Collins, C., Hewson, D. L., Munger, R., \& Wade, T. (2010) Evolving Models of Behavioral Health: Integration in Primary Care. New York: Milbank Memorial Fund.

Dekker, J. (2008) Theories in Behavioral Medicine. International Journal of Behavioral Medicine, 15, 1-3.

Denollet, J. (1993) Emotional distress and fatigue in coronary heart disease: the Global Mood Scale (GMS). Psychological Medicine, 23, 111-121.

Denollet, J., 2005. DS14: standard assessment of negative affectivity, social inhibition, and type D personality. Psychosomatic Medicine, 67, 8997.

Denollet, J., \& De Vries, J. (2006) Positive and negative affect within the realm of depression, stress and fatigue: The two factor distress model of the Global Mood Scale (GMS). Journal of Affective Disorders, 91, 171-180.

Marks, D. F. (2009) How should psychology interventions be reported? Journal of Health Psychology, 14, 4, 475-489.

Mechner, F. (2008). Behavioral contingency analysis. Behavioral Processes, 78, 124-144.

Michie, S., Johnston, M., Francis, J., Hardeman, W., \& Eccles, M. (2008) From theory to intervention: Mapping theoretically derived behavioural determinants to behaviour change techniques. Applied Psychology: An International Review, 57, 660-680.

Pedersen, S. S., \& Denollet, J. (2006) Is Type D personality here to stay? Emerging evidence across cardiovascular disease patient groups. Current Cardiology Reviews, 2, 205-213.

Siegrist, J., Wahrendorf, M., Knesebeck, O., Jürges, H., Börsch-Supan, A. (2006) Quality of work, wellbeing, and intended early retirement of older employees-baseline results from the SHARE study. European Journal of Public Health, 17, 1, 62-68. 
Schüz, B., Sniehotta, F. F., Mallach, N., Wiedemann, A. M., \& Schwarzer, R. (2009) Predicting transitions from preintentional, intentional and actional stages of change. Health Education Research, 24, 1, 64-75.

van Dijk, G.M., Veenhof, C., Spreeuwenberg, P., Coene, N., Burger, B.J., van Schaardenburg, D., van den Ende C.H., Lankhorst, G.J., Dekker, J., on behalf of the CARPA Study Group. (2010) Prognosis of limitations in activities in osteoar- thritis of the hip or knee: a 3-year cohort study. Archives of Physical Medical Rehabilitation, 91, 58-66.

Watson, D., Clark, L.A., \& Tellegen, A. (1988) Development and validation of brief measures of positive and negative affect: the PANAS scales. Journal of Personality and Social Psychology, 54, 1063-1070. 